\title{
On the Analysis and Modelling of the Harmonized Consumer Price Indices of West African Economic and Monetary Union Member States
}

\author{
Joseph Koula ${ }^{1, *}$, Tagouelbe Tiho ${ }^{2}$, Adasse Christophe Chiapo ${ }^{3}$ \\ ${ }^{1}$ Department of Mathematics and Computer Science, National Polytechnic Institute Felix Houphouet-Boigny, Yamoussoukro, Cote d'Ivoire \\ ${ }^{2}$ Department of Agriculture and Animal Resources, National Polytechnic Institute Felix Houphouet-Boigny, Yamoussoukro, Cote d'Ivoire \\ ${ }^{3}$ Department of Management, Business and Applied Economics, National Polytechnic Institute Felix Houphouet-Boigny, Yamoussoukro, \\ Cote d'Ivoire
}

\section{Email address:}

koulajoseph16@gmail.com (J. Koula), tihotag@gmail.com (T. Tiho), chiapoadass@yahoo.fr (A. C. Chiapo)

*Corresponding author

\section{To cite this article:}

Joseph Koula, Tagouelbe Tiho, Adasse Christophe Chiapo. On the Analysis and Modelling of the Harmonized Consumer Price Indices of West African Economic and Monetary Union Member States. American Journal of Theoretical and Applied Statistics.

Vol. 9, No. 6, 2020, pp. 283-295. doi: 10.11648/j.ajtas.20200906.14

Received: October 23, 2020; Accepted: November 6, 2020; Published: November 19, 2020

\begin{abstract}
The major goal of this paper is a better understanding of the price dynamics of the eight West African Economic and Monetary Union (WAEMU) member states. More specifically, the study intends to find the best models with suitable forecasting power for the monthly Harmonized Consumer Price Indices (HCPI) of each of the WAEMU countries. Descriptive statistics and time series modeling approaches were applied to the HCPI base $100=2008$ series covering the period from January 1998 to December 2019. The analysis revealed that Guinea-Bissau had the highest average HCPI of 99.88 and Senegal the lowest of 93.73. Togo attained the highest HCPI of 119.60 and Benin the lowest of 71.54 over the period studied. The indices of Togo and Guinea-Bissau have the highest and the smallest variance of 225.56 and 79.60, respectively. All the indices have an upward trend and contain cyclical and seasonal components. Using the Box-Jenkins methodology and Expert Modeler of SPSS five types of outliers, i.e. additive, additive patch, transient, innovational and level change, have been detected and different SARIMA models were proposed. Bartlett's B-test detects significant periodic effects in the residuals of the models for Burkina-Faso and Côte d'Ivoire. The residuals of all the models have been declared Gaussian by Shapiro-Wilks and Jarque-Bera normality tests while those of Côte d'Ivoire fail the latest test for normality due to the discrepancy of their skewness with that of a normal distribution. Adequacy of the claimed models has been corroborated by adequate values of key fitting and predicting statistics and the non-significance of the paired t-test on the mean difference between the observed and the adjusted values. Thus SARIMA $(0,1,0)(0,1,1)_{12}$ model was found to best fit the HCPI for Burkina Faso, Côte d'Ivoire, Niger, Senegal and Togo; and the data for Benin, Guinea Bissau and Mali are found to be SARIMA $(3,1,0)(1,0,1)_{12}$, SARIMA $(0,1,0)(1,0,1)_{12}$ and SARIMA $(1,1,1)(0,1,1)_{12}$ process, respectively. The differences between the retained models raise doubts on the claimed objective of convergence of the economies of the WAEMU countries. Engle's Lagrange Multiplier test for autoregressive conditional heteroscedasticity (ARCH) reveals the homoscedasticity of the residuals of all the models but the one of Côte d'Ivoire. Thus, for better modeling of the index of Côte d'Ivoire, a GARCH model may be envisioned.
\end{abstract}

Keywords: Harmonized Consumer Price Index, SARIMA, WAEMU, Outliers

\section{Introduction}

Price indices constitute through their history and their forecasts essential elements in decision making, planning, and policy evaluation. The harmonized consumer price index (HCPI) adopted in 1996 by the West African 
Economic and Monetary Union (WAEMU) member states is no exception to this regard. Of Laspeyres type, this index measures the variations of the general price level for a basket of goods and services consumed by the households of the major city of each of the countries between the basic period and a current period. As any price index, the HCPI has three main domains of possible uses; namely economic, accounting, and social domains. Indeed, it can serve for instance as a measuring instrument of the inflation and indexation of income or as a deflator of macroeconomic aggregates such as the Gross Domestic Product. The convergence of inflation rates constituting a requirement in the WAEMU, this index is a convenient multilateral surveillance instrument.

The HCPI has three components which are core inflation, energy, and fresh products, and not transformed cereals. The first component also knows as underlying inflation represents the long-run trend in the price levels. It does not account for items subject to volatile prices such as energy and food. The latter includes the volatile and seasonal food items, in particular vegetables, fish products, fruits, and the not transformed cereals such as rice, corn, millet, and sorghum. It contributes substantially to the volatility of the average HCPI of the WAEMU countries [1]. The "energy" component which includes fuels and lubricants renders the HCPI sensitive to the effects of outer shocks such as fluctuations in oil prices.

Many research works were conducted on price indices and inflation in general and HCPI in particular. ARIMA models [2] were used to study the inflation rate and price indices [36]. Gigunku and al. proposed a $\operatorname{SARIMA}(0,1,0)(0,0,1)_{4}$ model to explain and forecast inflation in Kenya [4] whereas Habimana and al. suggested an $\operatorname{ARIMA}(4,1,6)$ model for prices index of Rwanda [6]. Akpanta and Okorie proved that the consumer price index of Nigeria over the period 19962013 fits a $\operatorname{SARIMA}(1,2,1)(0,0,1)_{12}$ model [7]. Toé used SARIMA structure to model inflations calculated from the HCPI of WAEMU countries [1].

A seasonal autoregressive integrated moving average (SARIMA) model should thus better suit the HCPI: Their computation takes into account local foodstuffs inclined to introduce seasonal effect; that is fluctuations of their availability thus their price due to the seasons and recurring in a regular pattern in one year or other time intervals.

With regard to the usefulness of the HCPI mentioned above, the main objective of the present study is to provide adequate models to better study the HCPI of the WAEMU member states and provide reliable forecasts.

More specifically, this article intends to (i) characterize the HCPI, (ii) identify the SARIMA models which fit the HCPIs at best, and (iii) evaluate their forecasting capacity. It is organized as follows: this introductory section containing a brief literature review is followed by a section presenting the data used as well as elements of the methodology. The third section containing the main results and their interpretation precede the conclusion.

\section{Data and Methodology}

\subsection{Data of the Study}

The study uses the Harmonized Consumer Prices Indices base $100=2008$ of the 8 WAEMU countries. The data for all the member countries except those of Guinea Bissau starts on January 1998; whereas the series for this last country which, on May 2nd, 1997, joined the Union created on January 10th, 1994 spans from July 2002 thru October 2018. In 2018, the countries started changing the base year to 2014 with the consequence of different ending months of the series. The structure of the indices has even been changed. Thus, the data for Burkina-Faso and Togo go thru September 2018, the ones of Côte d'Ivoire and Senegal go thru December 2018. The data for Benin and Guinea Bissau end in October 2018 and those of Mali and Niger span thru April 2018 and August 2018, respectively. The 8 countries share borders in their great majority (Figure 1) and the Francs of French Community of Africa (FCFA) as common currency. The data were obtained from the official websites of the national statistical institutes and that of Afristat.

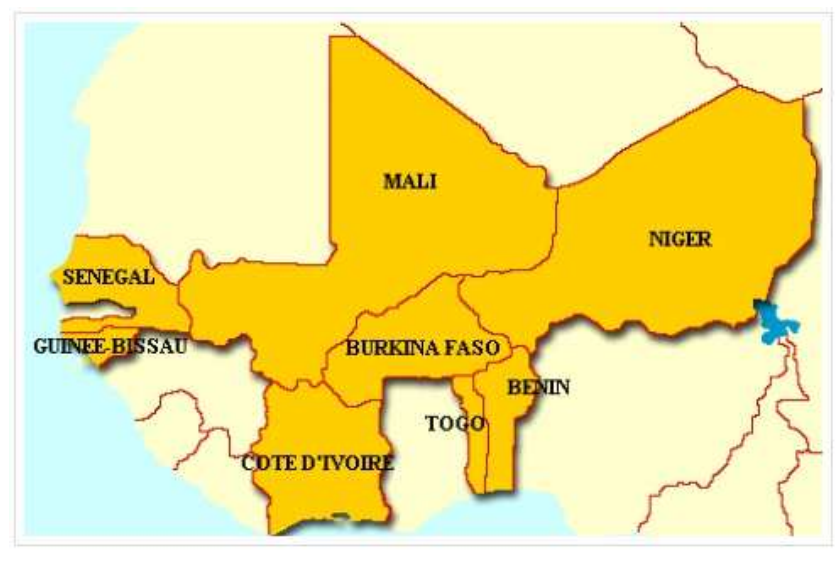

Figure 1. Map of the WAEMU member states.

\subsection{SARIMA Model}

In general, in time series analysis, in the presence of seasonal components, the effects of shocks are transmitted between times separated by $\mathrm{S}$ periods by an ARIMA (P, D, Q) process of the following equation:

$$
\Phi_{s}\left(B^{s}\right) \nabla_{s}^{D} Y_{t}=\Theta_{s}\left(B^{s}\right) \varepsilon_{t}
$$

and these shocks follow an ARIMA (p, d, q) model such that

$$
\Phi(B) \nabla^{d} \varepsilon_{t}=\Theta(B) u_{t}
$$

where $\mathrm{S}$ is the seasonal period; $\mathrm{d}$ and $\mathrm{D}$ are, the minimum integration order of the non-seasonal and seasonal component; respectively; $\mathrm{B}$ is the lag or backward shift operator such that $B^{k} y_{t}=y_{t-k} ; \nabla^{d}=(1-B)^{d}$ is the difference operator of order d; $\nabla_{S}^{D}=\left(1-B^{S}\right)^{D}$, the seasonal difference operator of order D. The $\left\{u_{t}\right\}$ series is white noise, that is to say independently (i.e. uncorrelated) and identically distributed with zero mean and constant variance $\sigma^{2}$. These noises may be Gaussian or not. $\mathrm{p}$ and $\mathrm{P}$ are the orders of non- 
seasonal and seasonal autoregressive processes, respectively. They indicate the number of past values of the series that allow the prediction of its present value. $\mathrm{q}$ and $\mathrm{Q}$ are, respectively, the orders of a non-seasonal and a seasonal moving average process. They indicate how many deviations from the mean of the past values of the series are taken into account in the formation of contemporary value.

The model obtained for $w_{t}=\nabla^{d} \nabla_{s}^{D} Y_{t}$ by multiplicatively combining the seasonal and non-seasonal components, by substituting the expression of $\varepsilon_{t}$ from (1) in (2) and taking into account a constant term $\delta$ is called a multiplicative Seasonal Autoregressive Integrated Moving Average model of order p, d, q, P, D, Q and $\mathrm{s}$ and denoted $\operatorname{SARIMA}(p, d, q)(P, D, Q)_{s}$ model. Its general equation is of the form:

$$
\Phi(B) \Phi_{s}\left(B^{s}\right) \nabla^{d} \nabla_{s}^{D} Y_{t}=\delta+\Theta(B) \Theta_{s}\left(B^{s}\right) u_{t}
$$

The constant $\delta=\Phi(B) \Phi_{S}\left(B^{S}\right) \mu_{w}$ with $\mu_{w}$ the average of $w_{t}$ can be kept in the model if the mean of the series is nonzero and to account for the possibility of a deterministic trend [8]. Its exclusion is often recommended when differentiating. The polynomials $\Phi, \Phi_{s}, \Theta, \Theta_{s}$ in B are defined as follows [9]:

$$
\begin{gathered}
\Phi(B)=1-\phi_{1} B-\phi_{2} B^{2}-\cdots-\phi_{P} B^{p} \\
\Phi_{S}\left(B^{S}\right)=1-\varphi_{1} B^{S}-\varphi_{2} B^{2 S}-\cdots-\varphi_{P} B^{P S} \\
\Theta(B)=1-\theta_{1} B-\theta_{2} B^{2}-\cdots-\theta_{q} B^{q} \\
\Theta_{S}\left(B^{S}\right)=1-\vartheta_{1} B^{S}-\vartheta_{2} B^{2 S}-\cdots-\vartheta_{Q} B^{Q S}
\end{gathered}
$$

where $\phi_{i}, \varphi_{j}, \theta_{k}$ et $\vartheta_{l}$ are such that the stationarity and the invertibility of the entire model are guaranteed. Equations (4) and (6) are respectively the non-seasonal autoregressive and moving averages operators and equations (5) and (7) are their seasonal equivalents.

\subsection{Modeling Approach}

The present study rests fundamentally on the methodology of Box-Jenkins; that is the modeling of a SARIMA process through the triptych identification-estimation of the parameters-diagnostic checking for validation of the model. These three steps can possibly be preceded by one of checking and establishing stationarity or followed by a step of forecast. The graphs of the initial times series, the correlograms, and other graphs of the residues can be of an essential recourse for a better understanding of stylized facts, the appreciation of the stationarity, and the validation of the proposed models. Checking for outliers and taking corrective measures if necessary, can considerably improve the resulting models. The use of adequate software substantially eases the implementation of this approach.

\subsubsection{Stationarity and Integration}

The methodology of Box-Jenkins requires the stationarity of the series; that is their mean, variance and, autocorrelation need to be constant at any instant $t$. To guarantee the reliability of the statistical inferences and forecast procedures, any time series need to be made stationary before being modeled [10]. Non-stationarity can be due to the presence of a trend, the heteroscedasticity of the series, the presence of a break in the dynamic of the series, etc.

In the presence of breaks in the trend, the power of the usual tests of stationarity such as those of Dickey-Fuller and Phillips-Perron are reduced [11]. Tests integrating this anomaly were proposed $[12,13]$ and incorporated into some econometric software. So, in setting up any SARIMA model, it is not only recommended to check for possible violation of the hypothesis of stationarity but also and above all to know the real causes and to remedy it, if necessary.

In case of non-stationarity of a time series $Y_{t}$, filters $(1-B)$ and $\left(1-B^{S}\right)$ can be used through the integration process to render $(1-B)^{d}\left(1-B^{S}\right)^{D} Y_{t}$ stationary. If necessary, the variance can be stabilized through a Box-Cox type of transformation of $Y_{t}$ that may be logarithmic or else.

\subsubsection{Outliers}

Extreme values can cause bad model specification, biases in parameter values, erroneous forecasts, and inappropriate decomposition [14] as well as non-stationarity of a time series. Their detection and, if necessary, corrective actions are thus advisable before any test of stationarity.

Four types of outliers are traditionally considered; that are additive, innovational, transient, and level shift [15-17]. An additive outlier impacts a single observation; the series then returns to its normal trajectory as if the shock had never occurred. An innovational extreme value produces a shock that propagates in the subsequent observations with the weightings scheme of a moving average representation of an ARIMA model [14]. The shock affects several observations if the series is stationary and can affect every observation from a particular point, otherwise [18]. The presence of an extreme value of level shift type is similar to step functions generated by a structural change [19]. Transient shocks are characterized by a peak, the impact of which decreases exponentially according to a constant factor of change until it disappears.

None of the above-mentioned types of extreme values is suited to capture solely the breaks in the trend of the seasonal component [14]. It is indeed admitted that the additive and transient extreme values affect the residual component, whereas the level change type of outliers concerns the trendcycle component and that those of innovational nature have a link with the seasonal and the trend-cycle components of a time series. So, it is essential to introduce in the typology the seasonal additive type which takes into account the disturbances solely in connection with the seasonal component. This type of outliers affects an observation in particular and in the same way the following observations separated by one or several seasonal periods [18].

Two other types of extreme values may also be considered: the local trend which appears at a specific point of the series and additive patch which concerns a group of at least two consecutive additive outliers.

\subsubsection{SARIMA Models: Identification and Estimation}

Identification of a SARIMA model consists of determining 
$\mathrm{p}, \mathrm{d}, \mathrm{q}, \mathrm{P}, \mathrm{D}$, and $\mathrm{Q}$, integers which characterize this process that can be additive or multiplicative. A multiplicative SARIMA is likely when the graph of the series suggests proportionality of the seasonal effect with the average of the series, that is the trend; or if the seasonal variation increases in time. On the other hand, if the seasonal effect is constant around the trend, an additive model should be more appropriate. In the presence of seasonality, the search for a "good" model must begin with a multiplicative SARIMA model before looking at additive models if necessary [2]. A logarithmic transformation of the time series will make the seasonal effect additive and therefore an additive SARIMA might be better indicated [20]. In any case, none of the two types of model should be ignored in the process.

The orders p, d, q, P, and Q can be suggested by an always not obvious examination of the graphs of the autocorrelation or the partial autocorrelation functions. Several SARIMA models with various values of these orders must be often compared. Parsimonious models, that is, models with a small number of parameters make unanimity among the specialists.

Several methods exist for the estimation of the SARIMA models. The presence of the white noise residual in the model requires a non-linear iterative procedure such as the maximum likelihood method.

In the Box-Jenkins, approach any model producing random or white noise residues is suited. Thus, several SARIMA models can compete and therefore other criteria need to be used to make a rational choice. The coefficients of determination $\mathrm{R}^{2}$ and stationary $\mathrm{R}^{2}$ can be used: the bigger in absolute value they are the better will the model be. In the presence of a trend or of a seasonal component, the second is preferable. It compares the stationary part of the model with a simple average model, i.e. $\operatorname{ARIMA}(0, d, 0)(0, D, 0) \cdot R^{2}$ is more useful when the model is stationary. Both $\mathrm{R}^{2}$ can be negative with a range of negative infinity of 1 . A negative (positive) value suggests that the proposed model is worse (better) than the basic model [18]. Other fit measures such as the root mean square error (RMSE), the mean absolute error (MAE), and the normalized Bayesian Criterion of Information (BIC) help in appreciating the global quality of models having the same explained variable. The smaller they are the better the model will be.

\subsubsection{SARIMA Models: Diagnosis, Validation, and Forecasting}

An adequate model must be able to reconstitute, explain, or predict at best the behavior of the process under study. The diagnosis and the validation of an ARIMA family of models rest essentially on the characteristics of the residues $\left\{\hat{u}_{t}\right\}$. Indeed, if the estimation of the model in equation (3) is suitable, $\left\{\hat{u}_{t}\right\}$ the unexplained component should not be able to significantly contribute to the explanation of the process under investigation. In other words, $\left\{\hat{u}_{t}\right\}$ has to be white noise, eventually Gaussian.

For a SARIMA model to be valid, the autocorrelations and the partial autocorrelations of the series of residues should not be statistically different from zero; if the autocorrelations of order 1 or 2 are significantly different from 0 , then the specifications ( $p, d, q)$ and $(P, D, Q)$ of the SARIMA model are probably unsuitable. However, one or two autocorrelations of upper order can accidentally exceed by chance the limits of the confidence intervals. This can relatively easily be appreciated through the graphs of residual autocorrelation and partial residual autocorrelation functions.

Besides, residues for "a good model" must be proved independent by a statistical test. For long data series, the white noise portmanteau test based on Box-Pierce Q statistic can be used. Ljung Q statistic, an adjustment of Box-Pierce Q for series of small size without altering his performances for the large samples exists. It can also be necessary to test deviations of a theoretical white noise process caused by periodic effects by using a test based on the spectra [21]. The Bartlett B test based on the cumulative periodogram is used in the present study to verify whether or not the series of residues contains significant periodicities. The nullity of the mean of the residues can be formally tested by a Student ttest and the normality of residues by a test such as that of Shapiro-Wilks. The constant nature of the variance of residues can be appreciated by a Lagrange Multiplier test equivalent to a $\chi^{2}$ which assesses the hypothesis of absence of autoregressive conditional heteroscedasticity (ARCH) effect against that of its presence.

\subsection{Data Analysis}

For a better description and characterization of the HCPIs, exploratory data analysis tools were used to produce graphics, statistics such as means, variance, and skewness.

The Expert Modeler of Statistical Package for Social Science (SPSS) was used to identify, estimate and select the best SARIMA model for the process of generation of the HCPI; this, for a set of specified extreme values. For data set without missing values like the one used, SPSS implements Melard's fast maximum likelihood algorithm [9] to estimate $\phi_{i}, \varphi_{j}, \theta_{k}$ and $\vartheta_{l}$, the parameters in equations (4) thru (7). This SPSS module makes the series stationary if necessary and automatically produces the most appropriate SARIMA model after detecting and taking into account the extreme values when required [9]. It eases the implementation of the Box-Jenkins approach and renders the correlograms and other graphs, checking tools rather than decision tools.

For statistical validation tests of the models, their residues were transferred in Stata. Once validated, the models have been used to generate forecasts under SPSS which uses either conditional or unconditional least squares [9]. Excel and SPSS were used to generate relevant graphs.

\section{Empirical Results and Interpretation}

\subsection{Characterization of the HCPIs}

Time series are often supposed to have four components: a trend, that represents the long-term evolution; a cyclical component which is a smooth, almost periodic movement around the trend with phases of growth and recession; a seasonal component representing the sub-annual fluctuations that are repeated more or less regularly from year to year; 
and an irregular component, regrouping all the more or less erratic fluctuations not taken into account in previous components. The trend and cycle components are often grouped as a trend-cycle component. For their adequate characterization, it seems necessary to at least make a general description of the HCPI and have some idea of the presence, nature and, if possible, the magnitude of their different potential components mentioned earlier.

\subsubsection{General Description of the HCPI}

The HCPIs of the eight WAEMU countries have a common global upward dynamic trend with essentially two phases. The first phase goes from January, 1998 to about the third quarter of 2008 whereas the second range from the first quarter of 2009 to the end of the series; that is the last quarter of 2019 (Figure 2). These phases are separated by a short transition period in the last quarter of 2008 .

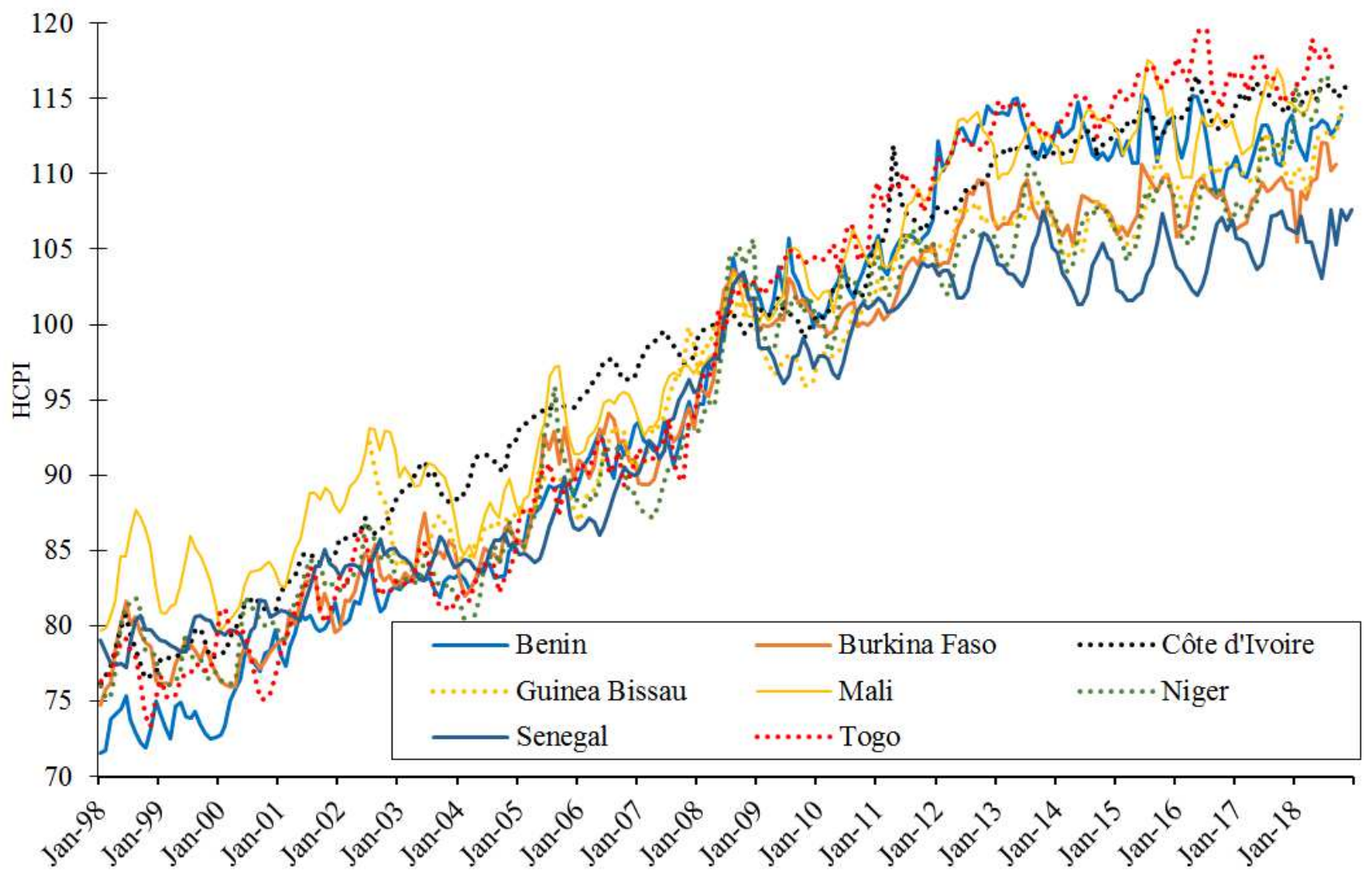

Months

Figure 2. Dynamic of monthly HCPI countries (01:1998-12:2018).

which is characterized by sharp rises in prices which then converge towards a single value about October 2008 and then slightly deflate thereafter. This constriction zone that can be assimilated to a point of structural change, coincides with the 2007-2008 global food crisis and the change of base year of the series from $100=1996$ to $100=2008$. The first phase mentioned above is characterized by a band of positive slope of more or less constant width. In the second phase, the indices are wrapped in a funnel-like band, limited downward by the HCPI of Senegal and upward by that of Togo. It tends to widen over time, thus suggesting price divergence from one month to the next within the WAEMU. In this phase, as of the first quarter of 2012; approximately two groups of HCPI emerge: a group of low-cost countries composed of Burkina Faso, Guinea Bissau, Niger, Senegal, and a group of high-cost countries constituted by Togo, Mali, Cote d'Ivoire, and Benin. Benin seems to have joined the first group in July 2016. Togo's HCPI consistently ranked among the lowest in the first phase became the highest after the last quarter of 2008.

From January 1998 to December 2018, the HCPIs of the eight countries differed more or less in their position, dispersion, shape, and flatness statistics. Togo, Mali, Côte d'Ivoire, and Niger had the highest average HCPIs (Table 1). HCPI averages, which range from 93.90 for Senegal to 99.95 for Guinea-Bissau, provide an indication of what HCPIs would have been had prices remained constant over the study period [7]. The HCPI of Togo, Benin, Côte d'Ivoire, Niger, Burkina Faso, and Mali have the highest variances, in decreasing order.

The skewness of the HCPI for all the countries are more or less symmetric: they are between -0.50 and +0.50 .

Based on the value of the kurtosis obtained using Stata, the distribution of the HCPI's are all flatter than a normal distribution which kurtosis is 3 this with negative excess kurtosis slightly lesser than -1 as confirmed by SPSS (Table 1). 
Table 1. Basic statistics of the HCPI of the WAEMU member states (01:1998-12:2018).

\begin{tabular}{llllllllll}
\hline \multirow{2}{*}{$\begin{array}{l}\text { Country } \\
\text { Number of } \\
\text { observations }\end{array}$} & Minimum & Maximum & Median & Mean & Variance & Skewness & Kurtosis & Value & Excess \\
\hline Benin & 250 & 71.54 & 115.24 & 99.14 & 96.09 & 209.10 & -0.18 & 1.53 & $-1,48$ \\
Burkina-Faso & 249 & 74.73 & 112.10 & 97.85 & 95.06 & 137.21 & -0.21 & 1.51 & $-1,49$ \\
Côte d'Ivoire & 252 & 76.16 & 116.50 & 99.80 & 98.88 & 156.58 & -0.24 & 1.77 & $-1,23$ \\
Guinea-Bissau & 196 & 83.68 & 114.60 & 100.97 & 99.94 & 80.13 & -0.35 & 1.73 & $-1,26$ \\
Mali & 244 & 79.59 & 117.60 & 97.58 & 99.01 & 133.59 & 0.00 & 1.57 & $-1,44$ \\
Niger & 248 & 75.12 & 116.50 & 96.15 & 94.99 & 142.38 & -0.08 & 1.54 & $-1,46$ \\
Senegal & 252 & 77.23 & 107.60 & 96.93 & 93.89 & 100.73 & -0.19 & 1.42 & $-1,59$ \\
Togo & 249 & 73.29 & 119.60 & 99.65 & 97.75 & 225.56 & -0.05 & 1.42 & $-1,59$ \\
\hline
\end{tabular}

\subsubsection{Trend and Cycle}

The Hodrick-Prescott high-pass filter is used in this study to separate the HCPI into trend and cyclical components. All the indices are characterized by upward long-term non-linear trends (Figure 3).

The non-linearity of the trend of the index of Côte d'Ivoire is less marked, unlike the ones of the other countries characterized by noticeable points of inflection implying a change of concavity that may be caused by an important economic change or shock. Most of those points occurred in the period 2007-2008. These trends components may contain a deterministic or a stochastic part. Cyclical components which contained short term trend and other fluctuations are present in the HCPI of all the eight WAMEU countries and seem to significantly explain the oscillatory movement of the HCPIs around their trends.

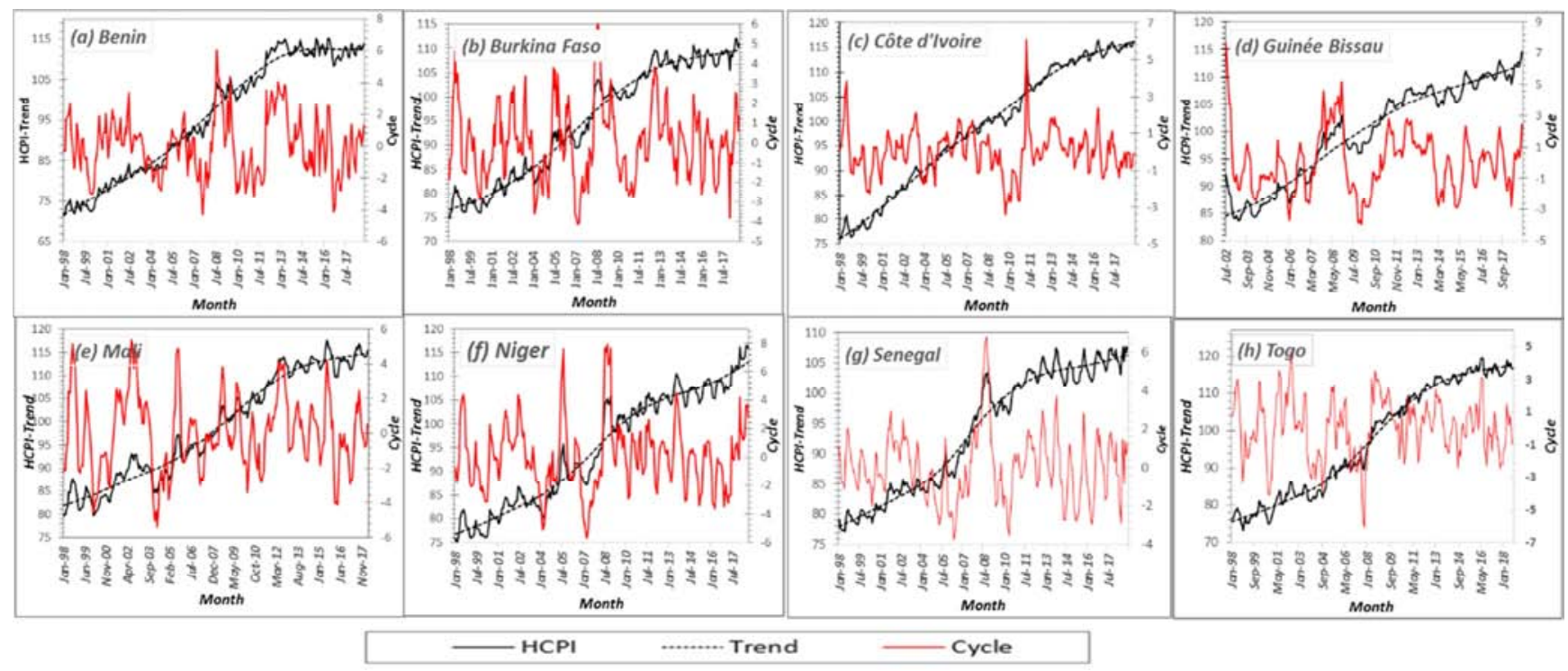

Figure 3. HCPI, cyclical and trend (Hodrick-Prescott filter) components of WAEMU states.

\subsubsection{Seasonal Component}

Seasonality in the HCPIs anticipated on the basis of the incorporation of seasonal products such as fresh products and unprocessed cereals in the computation of the indices is confirmed by the examination of the plots of series (Figures 2 and 3). It appears that each of the series has a pattern that repeats every $\mathrm{k}=12$ elements. The production and hence supply and prices of those products depend on seasons which may vary from one country of the Union to another or for the same country from one year to another. This is also established by the plots of the residual autocorrelation (ACF) and partial autocorrelation (PACF) functions of the initial HCPI with a seasonal and non-seasonal differentiation of orders 1 which show significant negative peaks at lags 12 and multiple of 12 (Figures 4 and 5) thereby suggesting a seasonal moving average component [3]. The opposite would have suggested a seasonal autoregressive component [22].

In 2011, seasonality accounts for about half of the volatility of the Union index and explains about $2 / 3$ of the IHPC volatility in Burkina Faso, Guinea-Bissau, and Senegal [1]. 

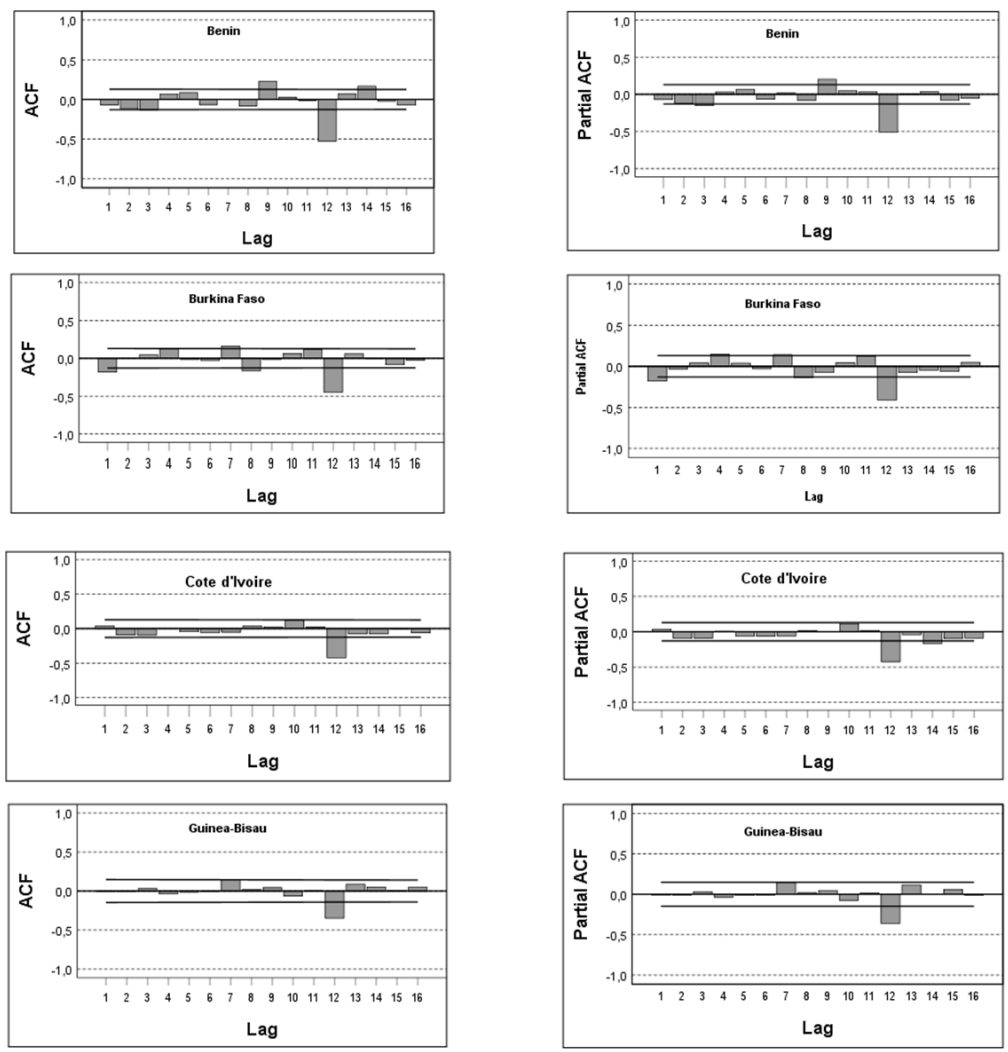

Figure 4. HCPI Autocorrelation functions for Benin, Burkina Faso, Cote d'Ivoire and Guinea-Bissau with seasonal and non-seasonal differentiation of order $D=1$ and $d=1$.
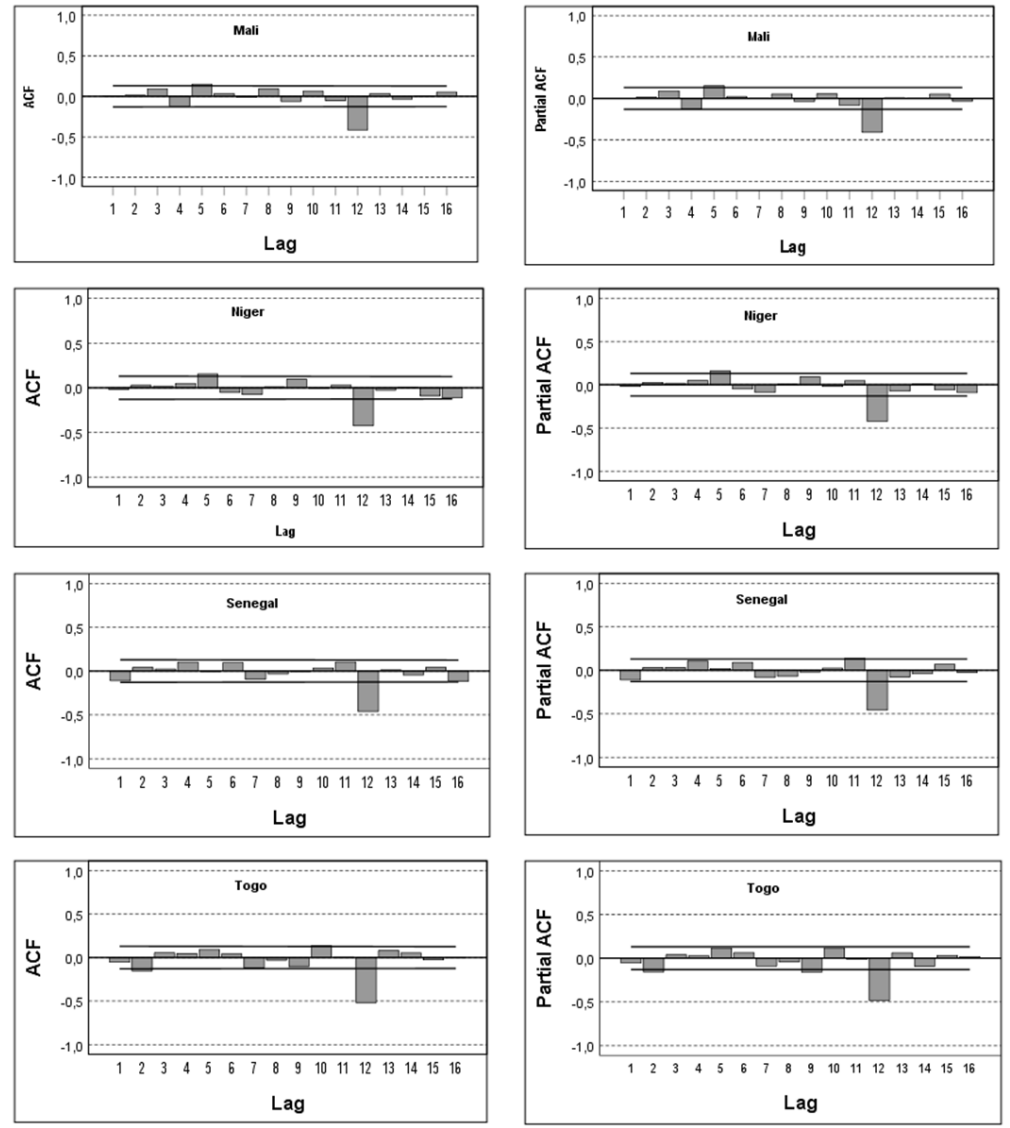

Figure 5. HCPI Autocorrelation functions for Mali, Niger, Senegal, and Togo with seasonal and non-seasonal differentiation of order D=1 and d=1. 


\subsubsection{Extreme Values}

The analysis reveals the presence of dated extreme values (Table 2), most of which can be assimilated to breakpoints corresponding to shocks of various kinds and origins, with effects of variable amplitudes and lengths. The extreme values identified are of additive, seasonal additive, additive patch, transient, innovational, and level change types. No local trend outliers have been detected.

Over the period covered by the study, events with significant impacts on the HCPIs took place in all WAEMU countries. This is the case, for example, with the economic and financial crisis from 2007 to 2008 and especially with the food crisis over the same period exacerbated by the spiraling rise in oil prices, which seem to have had the most impact and a diversity of effects on prices in the WAEMU zone: out of 33 extreme values detected 15 , i.e. $45.45 \%$, occurs over the period 2007-2009. These shocks, among others, may have contributed to the presence of transient effects in Guinea Bissau (January 2007) and Senegal (June 2008); level change effect in Benin (July 2008), Burkina (January 2008), Niger (July 2008, January 2009); and additives or additive patch effects in Benin, Guinea-Bissau, and Togo. Prices seem not to have been impacted by these crises in Côte d'Ivoire and Mali. Specific events have generated outliers in certain countries, such as Côte d'Ivoire, where, in November 2004, an atypical increase in the HCPI, corresponding to the resumption of hostilities in the military-political crisis that began in September 2002. Additive (April 2011) and innovational (June 2011) extreme values were also noted at the end of the post-electoral crisis period from December 2010 to April 2011 in that country.

Table 2. Extreme values of the HCPI of the WAEMU states (01:1998-12:2018).

\begin{tabular}{|c|c|c|c|c|}
\hline Country (number of outliers) * & Date & Type & & Estimation \\
\hline \multirow{6}{*}{ Benin (5) } & July 2008 & Level change" & & $3.468 * * *$ \\
\hline & \multirow{2}{*}{ August 2008} & \multirow{2}{*}{ Transient } & Magnitude & $3.239 * * *$ \\
\hline & & & Alteration factor & $0.626^{*}$ \\
\hline & June 2009 & Additive & & $-3.137 * * *$ \\
\hline & January 2012 & Level change & & $5.163 * * *$ \\
\hline & June 2015 & Innovational & & $4.513 * * *$ \\
\hline \multirow{2}{*}{ Burkina Faso (2) } & January 2008 & Level change & & $3.165 * * *$ \\
\hline & January 2018 & Additive & & $-2.834 * * *$ \\
\hline \multirow{3}{*}{ Côte d'Ivoire (3) } & November 2004 & Innovational & & $1.908 * * *$ \\
\hline & April 2011 & Additive & & $3.862 * * *$ \\
\hline & June 2011 & Innovational & & $-2.000 * * *$ \\
\hline \multirow{5}{*}{ Guinea Bissau (3) } & \multirow{2}{*}{ November 2007} & \multirow{2}{*}{ Transient } & Magnitude & $3.054 * * *$ \\
\hline & & & Alteration Factor & $0.594 * * *$ \\
\hline & January 2008 & Additive & & $-2.097 * * *$ \\
\hline & \multirow{2}{*}{ November 2008} & \multirow{2}{*}{ Transient } & Magnitude & $3.192 * * *$ \\
\hline & & & Alteration Factor & $0.591 * *$ \\
\hline \multirow{3}{*}{ Mali (3) } & October 2005 & Innovational & & $-3.008 * * *$ \\
\hline & January 2011 & Additive & & $1.900 * * *$ \\
\hline & January 2016 & Innovational & & $-3.013 * * *$ \\
\hline \multirow{5}{*}{ Niger (4) } & August 2005 & Additive & & $2.575 * * *$ \\
\hline & July 2008 & Level change & & $5.405^{* * *}$ \\
\hline & January 2009 & Level change & & $-5.277 * * *$ \\
\hline & \multirow{2}{*}{ January 2018} & \multirow{2}{*}{ Transient } & Magnitude & $4.825^{* * *}$ \\
\hline & & & Alteration factor & $0.855^{* * *}$ \\
\hline \multirow{5}{*}{ Senegal (4) } & October 2005 & Additive & & $1.505^{* * *}$ \\
\hline & \multirow{2}{*}{ June 2008} & \multirow{2}{*}{ Transient } & Magnitude & $2.725 * * *$ \\
\hline & & & Alteration Factor & $0.800 * * *$ \\
\hline & January 2009 & Innovational & & $-2.899 * * *$ \\
\hline & September 2018 & Additive & & $-2.561 * * *$ \\
\hline \multirow{9}{*}{ Togo (9) } & July 2001 & Additive & & $1.596^{*}$ \\
\hline & August 2001 & Additive & & $1.454^{*}$ \\
\hline & July 2006 & Level change & & $-2.066 * *$ \\
\hline & September 2006 & Seasonal Additive & & $0.642 *$ \\
\hline & August 2007 & Additive & & $-2.877 * * *$ \\
\hline & September 2007 & Additive & & $-3.367 * * *$ \\
\hline & October 2007 & Additive & & $-3.296 * * *$ \\
\hline & May 2008 & Additive & & $2.152 * * *$ \\
\hline & August 2016 & Level change & & $-3.632 * * *$ \\
\hline
\end{tabular}

Level of significance: ***: p-value $<0.001$; **: p-value $<0.01$; * p-value $<0.05$ 
The unusual rise in the consumer price index in Benin in an innovational manner in January 2012 is mainly due to higher prices for transport services caused by fuel price pressure following the reduction by $50 \%$ of subsidies on petroleum product prices in neighboring Nigeria. Cereals have often been the sources of the drastic rise in prices, especially in the Sahelian countries such as Burkina, Mali, Niger, and Senegal. The large number of shocks i.e. 9 on the prices for Togo causes the most important variance of 225 among all the $8 \mathrm{HCPI}$ studied. The HCPI of Benin, a neighbor of Togo, impacted over the period of the study by 5 shocks had the second-highest variance as indicated in Table 1.

\subsection{SARIMA Models for the HCPI of the WAEMU Member States}

\subsubsection{Fitted and Proposed Models}

After estimation and comparison of different SARIMA models, the lag and forecasting forms as well as the fitting quality indicators of those retained are shown in Table 3.

Over the periods covered by the study, the HCPI of Burkina Faso, Côte d'Ivoire, Niger, Senegal and Togo have no regular and no seasonal autoregressive; no regular moving average and one seasonal moving average parameters. Their parameters were estimated after they were differenced once with lag 1 , and once seasonally differenced. Thus, they are generated by a SARIMA $(0,1,0)(0,1,1)_{12}$ process. The HCPI of Benin follows a SARIMA $(3,1,0)(1,0,1)_{12}$ model whereas those of Guinea Bissau and Mali are generated by SARIMA $(0,1,0)(1,0,1)_{12}$ and SARIMA $(1,1,1)(0,1,1)_{12}$ models, respectively. Thus, the models of all the countries have a seasonal moving average component. Only those of
Benin and Guinea Bissau contain a seasonal autoregressive parameter. Regular moving average and autoregressive components are simultaneously present in the HCPI of Mali while those of Benin contain three of the latest.

The similarity or not of the processes supporting the price formation in the different countries of the Union, may be explained inter alia by the sharing or not of common borders. This may explain the exact similarity of the coefficient of the expanded models for the HCPI of Burkina Faso, Côte d'Ivoire and to a lesser degree Niger. Other factors such as the intensity of trade between the states of the Union or between them and their neighboring non-Member States should not be neglected. For instance, Burkina Faso has in addition to Côte d'Ivoire and Togo, Ghana as a major trading partner. Benin has a very tied trade relation with Nigeria.

It is worth to note that except the models of Guinea-Bissau and Benin characterized by $\mathrm{P}=1, \mathrm{D}=0, \mathrm{Q}=1$ those of the other countries share the same seasonal structure; that is $\mathrm{P}=0, \mathrm{D}=1$, and $\mathrm{Q}=1$. This confirms the fact that the initial eight times series have a seasonal moving average component.

All the proposed models account for almost all of the total variability of the series $\left(R^{2} \geq 99 \%\right)$. The stationary $R^{2}$ which compares the stationary part of the model with a simple mean model being positive for all the models, their subconsiderations are better than the baseline SARIMA $(0, \mathrm{~d}, 0)$ $(0, \mathrm{D}, 0)$ model for all the countries: the proposed HCPI proposed outperforms the mean model by at least $25 \%$ for Guinea Bissau and at most $60 \%$ for Côte d'Ivoire and Togo. With the presence of a trend or a seasonal pattern in the HCPIs, the stationary $\mathrm{R}^{2}$ must be preferred to $\mathrm{R}^{2}$ to appreciate the quality of the fit.

Table 3. SARIMA models for HCPI of the West African Monetary and Economic Union member states.

\begin{tabular}{|c|c|c|c|c|c|c|}
\hline Country SARIMA Model & Model Formulations & Stationary $R^{2}$ & $R^{2}$ & RMSE & MAPE & NBIC* \\
\hline $\begin{array}{l}\text { Benin } \\
\operatorname{SARIMA}(3,1,0)(1,0,1)_{12}\end{array}$ & $\begin{array}{l}\dagger\left(1-0.195 B^{3}\right)\left(1-0.983 B^{12}\right)(1-B) Y_{t}=\left(1-0.906 B^{12}\right) u_{t} \\
\neq y_{t}=y_{t-1}-0.195\left(y_{t-3}-y_{t-4}\right)+0.983\left(y_{t-12}-y_{t-13}\right)+ \\
0.192\left(y_{t-15}-y_{t-16}\right)+u_{t}-0.906 u_{t-12}\end{array}$ & 0.365 & 0.996 & 0.950 & 0.799 & 0.096 \\
\hline $\begin{array}{l}\text { Burkina Faso } \\
\operatorname{SARIMA}(0,1,0)(0,1,1)_{12}\end{array}$ & $\begin{array}{l}\left(1-B^{12}\right)(1-B) Y_{t}=\left(1-0.861 B^{12}\right) u_{t} \\
\quad y_{t}=y_{t-1}+\left(y_{t-12}-y_{t-13}\right)+u_{t}-0.861 u_{t-12}\end{array}$ & 0.461 & 0.993 & 0.931 & 0.780 & -0.074 \\
\hline $\begin{array}{l}\text { Côte d'Ivoire } \\
\operatorname{SARIMA}(0,1,0)(0,1,1)_{12}\end{array}$ & $\begin{array}{l}\left(1-B^{12}\right)(1-B) Y_{t}=\left(1-0,867 B^{12}\right) u_{t} \\
y_{t}=y_{t-1}+\left(y_{t-12}-y_{t-13}\right)+u_{t}-0,867 u_{t-12}\end{array}$ & 0.601 & 0.998 & 0.535 & 0.424 & $-1,161$ \\
\hline $\begin{array}{l}\text { Guinea Bissau } \\
\text { SARIMA }(0,1,0)(1,0,1)_{12}\end{array}$ & $\begin{array}{l}\left(1-0.994 B^{12}\right)(1-B) Y_{t}=\left(1-0.942 B^{12}\right) u_{t} \\
y_{t}=y_{t-1}+0.994\left(y_{t-12}-y_{t-13}\right)+u_{t}-0.942 u_{t-12}\end{array}$ & 0.247 & 0.992 & 0.829 & 0.631 & -0.186 \\
\hline $\begin{array}{l}\text { Mali } \\
\operatorname{SARIMA}(1,1,1)(0,1,1)_{12}\end{array}$ & $\begin{array}{l}(1+0.885 B)(1-B)\left(1-B^{12}\right) Y_{t}=(1+0.995 B)\left(1-0.812 B^{12}\right) u_{t} \\
y_{t}=0.115\left(y_{t-1}-y_{t-13}\right)+0.885\left(y_{t-2}+y_{t-14}\right)+y_{t-12}+u_{t}+ \\
0.995 u_{t-1}-0.812 u_{t-12}-0.808 u_{t-13}\end{array}$ & 0.489 & 0.995 & 0.798 & 0.626 & -0.310 \\
\hline $\begin{array}{l}\text { Niger } \\
\operatorname{SARIMA}(0,1,0)(0,1,1)_{12}\end{array}$ & $\begin{array}{l}(1-B)\left(1-B^{12}\right) Y_{t}=\left(1-0,847 B^{12}\right) u_{t} \\
y_{t}=y_{t-1}+\left(y_{t-12}-y_{t-13}\right)+u_{t}-0.847 u_{t-12}\end{array}$ & 0.560 & 0.994 & 0.905 & 0,727 & $-0,061$ \\
\hline $\begin{array}{l}\text { Senegal } \\
\operatorname{SARIMA}(0,1,0)(0,1,1)_{12}\end{array}$ & $\begin{array}{l}(1-B)\left(1-B^{12}\right) Y_{t}=\left(1-0,831 B^{12}\right) u_{t} \\
y_{t}=y_{t-1}+\left(y_{t-12}-y_{t-13}\right)+u_{t}-0.831 u_{t-12}\end{array}$ & 0.560 & 0.996 & 0.613 & 0.509 & -0.840 \\
\hline $\begin{array}{l}\text { Togo } \\
\operatorname{SARIMA}(0,1,0)(0,1,1)_{12}\end{array}$ & $\begin{array}{l}(1-B)\left(1-B^{12}\right) Y_{t}=\left(1-0.911 B^{12}\right) u_{t} \\
y_{t}=y_{t-1}+\left(y_{t-12}-y_{t-13}\right)+u_{t}-0.911 u_{t-12}\end{array}$ & 0.601 & 0.997 & 0.856 & 0.668 & -0.080 \\
\hline
\end{tabular}

$\dagger$ : Compact expression; $\neq$ : Forecasting or expanded equation; *: Normalized BIC

\subsubsection{Diagnostic and Validation of the Models}

The residuals of the proposed models for the HCPI of the 8 WAEMU member states have all been declared white noises by Portmanteau and Ljung-Box (P-value $\geq 0.05$ ) Q tests. Therefore, the residuals are independently distributed. In other terms, there exists no serial correlation among the residuals. Moreover, the cumulative periodogram-based Bartlett's B-test detects significant periodic effects in the residuals of the models for Burkina-Faso (P-value=0.037) and Côte d'Ivoire $(\mathrm{P}$-value=0.015). The results of the tests for white noise residuals are recorded in Table 4. 
Table 4. Test for white noise residuals.

\begin{tabular}{lllllll}
\hline \multirow{2}{*}{ Modèle } & \multicolumn{2}{l}{ Portemanteau Q test } & Ljung-Box Q test & \multicolumn{2}{l}{ Bartlett B test } \\
\cline { 2 - 7 } & $\mathbf{Q}$ & Prob $>\boldsymbol{\chi}^{\mathbf{2}}(\mathbf{4 0})$ & $\mathbf{Q}$ & Prob $>\boldsymbol{Q}(\mathbf{1 8})$ & B & Prob $>$ B \\
\hline Benin & 45.148 & 0.265 & 18.479 & 0.238 & 0.654 & 0.785 \\
Burkina Faso & 48.381 & 0.171 & 23.893 & 0.122 & 1.411 & 0.037 \\
Côte d'Ivoire & 45.763 & 0.245 & 24.243 & 0.113 & 1.567 & 0.015 \\
Guinea Bissau & 35.688 & 0.666 & 11.906 & 0.750 & 0.849 & 0.467 \\
Mali & 38.622 & 0.532 & 15.364 & 0.426 & 0.517 & 0.952 \\
Niger & 43.846 & 0.312 & 16.640 & 0.479 & 0.743 & 0.639 \\
Senegal & 33.535 & 0.860 & 15.421 & 0.565 & 0.622 & 0.834 \\
Togo & 35.862 & 0.657 & 26.597 & 0.064 & 1.122 & 0.161 \\
\hline
\end{tabular}

As indicated in Table 5, Student t-tests for the equality of residual mean to zero were not significant for all the models. The residual being the difference between the observed and the predicted values, at a given point in time, this reveals the good quality of the different models particularly for forecasting purpose.

Table 5. Student t-test for the equality to zero of the residual means.

\begin{tabular}{llll}
\hline Country & Mean & Student-t & $\boldsymbol{P}(|\boldsymbol{T}|>|\boldsymbol{t}|)$ \\
\hline Benin & 0.0258 & 0.4363 & 0.6630 \\
Burkina Faso & -0.0074 & -0.1230 & 0.9022 \\
Côte d'Ivoire & -0.0101 & -0.2973 & 0.7665 \\
Guinea Bissau & 0.0790 & 1.3754 & 0.1706 \\
Mali & 0.0366 & 0.7065 & 0.4806 \\
Niger & 0.0228 & 0.3913 & 0.0696 \\
Senegal & 0.0248 & 0.6397 & 0.5230 \\
Togo & 0.0266 & 0.4874 & 0.6264 \\
\hline
\end{tabular}

The Melard's algorithm used by SPSS for estimating ARIMA models which uses the Maximum Likelihood method assumes a Gaussian white noise [18]. The residuals of all the models but that of Côte d'Ivoire pass the JarqueBera skewness and kurtosis tests for normality (Pvalue $<0.05$ ). For that country, this test detects discrepancy of the skewness of the residuals with that of a normal distribution (P-value $<0,013$ ). Shapiro Wilks normality tests, more powerful regardless of the degree of asymmetry of the distribution [23] confirm the normality of residuals (Pvalue $\geq 0.05$ ) for all the models. The results of the tests for normality of the residuals are summarized in Table 6.

Table 6. Results of normality test for the residuals.

\begin{tabular}{|c|c|c|c|c|c|c|c|c|}
\hline \multirow{3}{*}{ Model } & \multicolumn{4}{|c|}{ Jarque Bera Skewness/Kurtosis test for normality } & \multicolumn{4}{|c|}{ Shapiro Wilks W test for normality } \\
\hline & \multirow{2}{*}{ Pr (Skewness) } & \multirow{2}{*}{ Pr (Kurtosis) } & \multicolumn{2}{|l|}{ Joint test } & \multirow{2}{*}{ W } & \multirow{2}{*}{$\mathbf{V}$} & \multirow{2}{*}{$\mathbf{z}$} & \multirow{2}{*}{ Prob $>z$} \\
\hline & & & Adjusted $\chi^{2}$ & Prob $>\chi^{2}$ & & & & \\
\hline Benin & 0.5755 & 0.5129 & 0.75 & 0.6879 & 0.9953 & 0.841 & -0.403 & 0.6567 \\
\hline Burkina Faso & 0.2870 & 0.7046 & 1.29 & 0.5249 & 0.9924 & 1.306 & 0.619 & 0.2679 \\
\hline Côte d'Ivoire & 0.0119 & 0.0637 & 8.89 & 0.0117 & 0.9881 & 2.069 & 1.688 & 0.0457 \\
\hline Guinea Bissau & 0.4591 & 0.4443 & 1.15 & 0.5639 & 0.9938 & 0.911 & -0.215 & 0.5851 \\
\hline Mali & 0.0889 & 0.4383 & 3.53 & 0.1716 & 0.9879 & 2.051 & 1.665 & 0.0480 \\
\hline Niger & 0.1780 & 0.2030 & 3.46 & 0.1769 & 0.9897 & 1.772 & 1.327 & 0.0923 \\
\hline Senegal & 0.5287 & 0.8897 & 0.42 & 0.8106 & 0.9951 & 0.849 & -0.379 & 0.6477 \\
\hline Togo & 0.9972 & 0.0697 & 3.32 & 0.1903 & 0.9919 & 1.382 & 0.750 & 0.2266 \\
\hline
\end{tabular}

Engle's Lagrange Multiplier test for the presence of conditional autoregressive heteroscedasticity (ARCH) reveals that the residual variances are constant for all the models with the exception of the model of Côte d'Ivoire (Table 7). Thus, for the SARIMA model for Côte d'Ivoire, even though the unbiasedness of the parameter estimate shouldn't be a problem because of the relatively large sample size there is no guarantee that its variance is minimum. The tests concerning those estimates might give a false sense of precision. 
Table 7. Lagrange multiplier tests for the presence or not of ARCH effect, lag (1) (HO: no ARCH effect).

\begin{tabular}{lll}
\hline Country & Chi2 & $\boldsymbol{P}>$ Chi2 \\
\hline Benin & 0.238 & 0.6257 \\
Burkina Faso & 0.410 & 0.5218 \\
Côte d'Ivoire & 20.993 & 0.0000 \\
Guinea Bissau & 0.050 & 0.8237 \\
Mali & 1.020 & 0.3125 \\
Niger & 0.299 & 0.5844 \\
Senegal & 0.068 & 0.7944 \\
Togo & 0.107 & 0.7438 \\
\hline
\end{tabular}

From the foregoing, the proposed SARIMA models for HCPIs in all UEMOA countries have white noise residuals. Apart from that of Côte d'Ivoire, all other models are characterized by Gaussian homoscedastic white noise residuals. These models are therefore all acceptable representations of the data. Nevertheless, it is worth mentioning that the HCPI for Côte d'Ivoire might be better modeled as being generated by a general autoregressive conditional heteroscedastic process which models the volatility itself. This will probably allow a more precise understanding of the up and down changes in the dynamic of the HCPI that might be expected during the next month (s).

\subsubsection{Forecasting Performances of the Models}

Despite the difference in the ending periods of the time series, for the sake of good ground of comparison of the forecasting performances of the models of the 8 countries, a common forecasting horizon, say 12-months, is here retained.

Based in part on model statistics such as RMSE, MAPE, and the normalized Bayesian Information Criterion (Table 3), SPSS Expert Modeler reported the proposed models as the best SARIMA models. The visual examination of the observed, adjusted and predicted values as well as the confidence band around these values in Figure 5 confirms the adequacy of these models as verified by the t-tests on the nullity of the residuals mean value (Table 5).

Unlike Akpanta and Okorie [7] who tested the equality to zero of the difference of the mean of the forecast and the mean of the observed values, the choice is here made to test the equality to zero of $\mu_{\text {Diff }}$, the mean of the difference between the forecast and observed values of the HCPI for each month. The results of these bilateral paired t-tests are identical to the one concerning the equality to zero of the mean of the residuals defined as the difference between the observed and the predicted value at each point in time (Table 5). They revealed no significant difference in the models of all the eight countries. This confirms the fact that there is no significant difference between the observed and the value predicted in each month by the different models. Thus, the models have good predicting powers. More one projects in the future more the precision of the forecasts decreases: the width of the band of confidence around the forecasts tends to increase with time (Figure 6). This corroborates the fact that ARIMA models place more emphasis on the recent past than on the distant one, making short-term forecasts more accurate than long-term ones [17, 21].

\section{Conclusion}

Parsimonious SARIMA models having white noise residuals, good fitting and forecasting abilities have been proposed as processes for generating the HCPI of each of the eight West African Economic and Monetary Union member countries. The HCPI of Burkina Faso, Côte d'Ivoire, Niger, Senegal, and Togo are found to be spawned by a SARIMA $(0,1,0)(0,1,1)_{12}$ process. The HCPI of Benin, Guinea Bissau and Mali are, respectively generated by a $\operatorname{SARIMA}(0,1,3)(1,0,1)_{12}$, a $\operatorname{SARIMA}(0,1,0)(1,0,1)_{12}$ and a SARIMA $(1,1,1)(0,1,1)_{12}$ process. These models, which take into account shocks that would have occurred on prices appear to be more efficient than those that ignore the extreme values that result from these shocks. Thus, detection and modeling of atypical values are suggested whenever possible in the modeling process of times series. With this approach, recourse to the major past economic, financial, and social events in the countries should allow more insight in the explanation of the fluctuations in the prices like structural models do. Despite the good behavior of the SARIMA model for Côte d'Ivoire, the fact remains that it is characterized by a problem of heteroscedasticity of the residues which could impact the efficiency of the parameters estimates. ARCH family modeling would certainly be more convenient. In addition, given the relatively large length of the series, has non-normality of the residuals been a problem the proposed model for Côte d'Ivoire as suggested by the Jarque-Bera test would not have disturbed the consistency of the estimates and the central limit theorem would have guaranteed valid inferences in an asymptotic sense.

The HCPI base 100=2008 being computed for a basket of items in the major cities of the country this tempers its ability to establish the correct relation between countries. This will probably be corrected by its new version base $100=2014$ which covers the whole country and is implemented since the second quarter of 2018 the WAEMU states. 

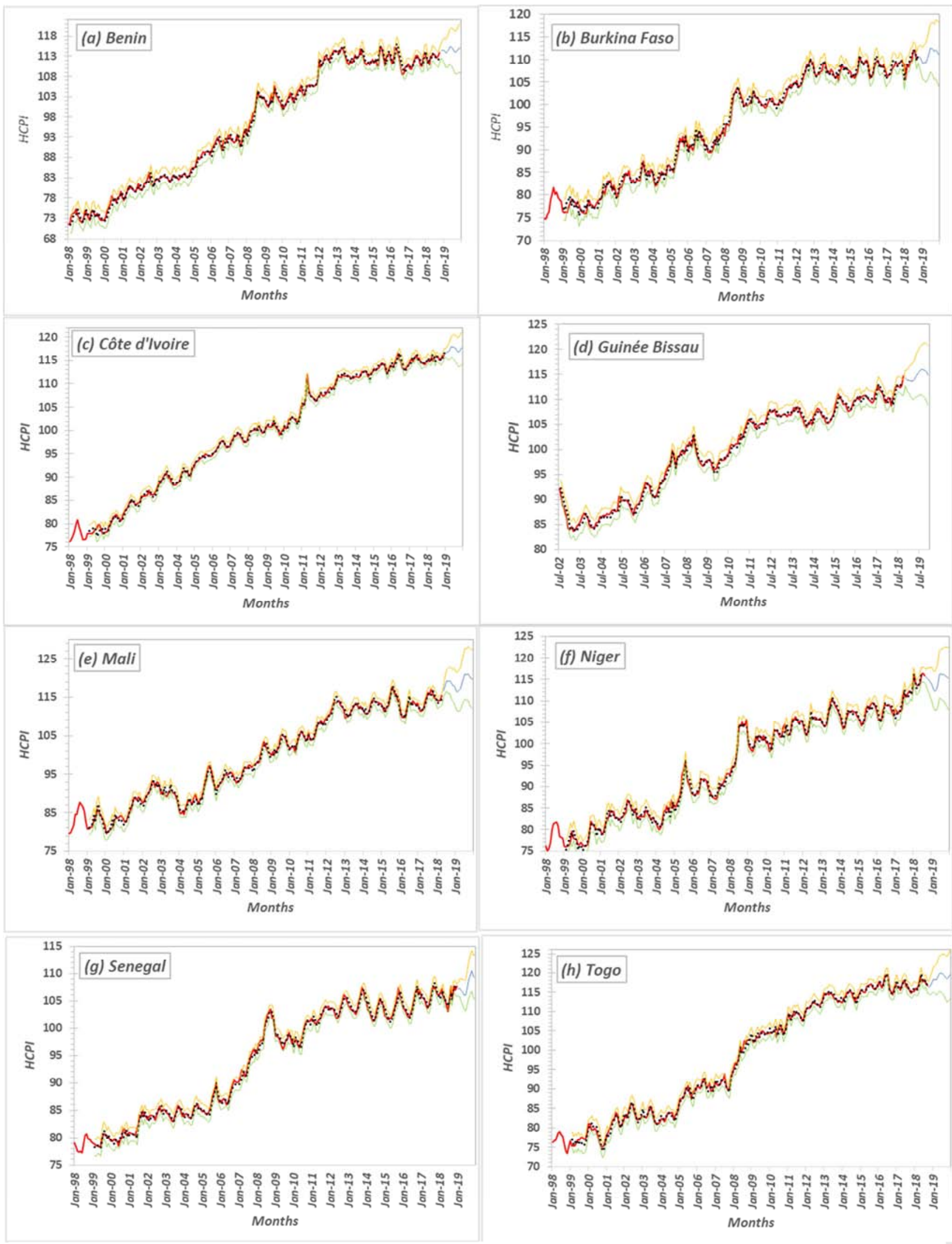


\section{References}

[1] D. M. Toé. Prévision de l'inflation dans la zone UEMOA: une approche par composantes. BCEAO. Dept. Etudes Economiques et de la Monnaie, 2011, $\mathrm{N}^{\circ} \mathrm{DER} / 11 / 02$.

[2] G. E. P. Box, G. M. Jenkins, and G. C. Reinsel, Time series analysis: forecasting and control. $4^{\text {th }}$ ed. Hoboken, NJ: Wiley, 2008 , p. 765.

[3] E. Aidoo. Modeling and forecasting inflation rates in Ghana: an application of SARIMA models, 2010, Unpublished Master Thesis for Degree in Applied Statistics, School of Technology and Business Studies, Hogskolan Dalarna.

[4] Gikungu, S. W., Waititu, A. G., and Kihoro, J. M (2015). Forecasting inflation rate in Kenya using SARIMA model. American Journal of Theoretical and Applied Statistics; 4 (1): 15-18.

[5] Etuk, H. E. (2012). Seasonal Arima Model to Nigerian consumer price index data. American Journal of Science and Industrial Research, 3 (5): 283-287.

[6] Habimana, N., Wanjoya, A., and Waititu, A. (2016). Modeling and Forecasting Consumer Price Index (Case of Rwanda). American Journal of Theoretical and Applied Statistics, 5 (3): 101-107.

[7] Akpanta, A. C. and Okorie, I. E. (2015) On the Time Series Analysis of Consumer Price Index data of Nigeria -1996 to 2013. American Journal of Economics 2015, 5 (3): 363-369.

[8] R. H. Shumway and D. S. Stoffer, Time Series Analysis and Its application, Springer, New-York, Berlin, 2000, p. 568.

[9] IBM, (2016). IBM SPSS Statistics 24 Algorithms. Copyright IBM Corporation 1989, 2016.

[10] Tsasa, J.-P. K. (2014) Test de racine unité et analyse des ruptures. Tendance stochastique, correction [non] paramétrique et stratégie de détection, Société congolaise d'économétrie, papier SCE 3, 81-111.

[11] Perron, P. (1989). The great crash, the oil price shock, and the unit root hypothesis, Econometrica, 57, 1361-1401.

[12] Clemente, J., Montañés A., and Reyes M. (1998). Testing for a unit root in variables with a double change In The mean, Economics Letters, 59, 175-182.

[13] Kapetanios, G. (2002), Unit root testing against the alternative hypothesis of up to m structural breaks, Working Papers 469, Queen Mary, University of London, Department of Economics.

[14] R. Kaiser, and A. Maravall, Seasonal outliers in time series. Banco d'Espana-Servicio de Estudios, Documento de Trabajo n' 9915, 2001, p. 37.

[15] Fox, A. J. (1972). Outliers in Time Series. Journal of Royal Statistics Society, B (34), 350-363.

[16] Chen; C., and Liu, L. (1993). Joint Estimation of Model Parameters and Outliers in Time Series, Journal of the American Statistical Association, 88, 284-297.

[17] D. Pena, Outliers, Influential Observations, and Missing Data, Chapter 6 in D. Peña, G. C. Tiao, and R. S. Tsay, A Course in Time Series Analysis, 2001, pp: 136-170.

[18] IBM, (2015). IBM SPSS Forecasting 24. Copyright IBM France 2015.

[19] Tsay, S. R. (1988). Outliers, Level Shifts, and Variance Changes in Time Series; Journal of Forecasting, 7, 1-20.

[20] C. Chatfield, The Analysis of Time Series: An Introduction. 6th ed. Boca Raton, FL: Chapman \& Hall/CRC, 2004, p. 338.

[21] G. M. Jenkins and D. G. Watts, "Spectral Analysis and Its Applications," Holden-Day, San Francisco, 1968, p. 525.

[22] Etuk, H. E. (2013). Multiplicative SARIMA modeling of daily naira -euro exchange rates. International Journal of Mathematics and Statistics Studies, 1 (3): 1-8.

[23] Yap, B. W., and Sim, C. H. (2011). Comparison of Various Types of Normality Tests. Journal of Statistical Computation and Simulation, 81 (12): 2141-2155. 